\title{
Passive acoustic monitoring and automated analysis as an alternative to trapping for monitoring species richness and seasonal swarming behaviour of Myotis bats
}

\author{
R Thomas ${ }^{1}$ and Stephen Davison ${ }^{2}$ \\ ${ }^{1}$ University of Cardiff \\ ${ }^{2}$ Affiliation not available
}

November 2, 2020

\begin{abstract}
1. Bat abundance, diversity and behaviour can be monitored by capturing bats for identification and measurement in the hand, but this has several disadvantages. These include disturbance to the bats, which limits the frequency with which captures can be made at an individual capture site, and potentially alters the behaviours being studied. 2. Passive recording and automated analysis and identification of bat calls offers an alternative, non-invasive approach to monitoring bats. In this study, we examine the effectiveness of acoustic monitoring in comparison with capture-based monitoring of seasonal swarming behaviour among several species of Myotis bats in southern Britain. 3. We show that both approaches have advantages and disadvantages for different tasks, but can be viewed as complementary methods for addressing different types of research questions. 4. We applied these complementary approaches, together with observations from infra-red video monitoring, to describe seasonal, overnight and species-specific variation in swarming behaviour in a multi-species community of Myotis bats. 5 . In our study of swarming behaviour, capture and examination of bats in the hand was necessary for measuring sex ratios, reproductive status, and even for confirmation of species identification for some difficult to separate taxa. Capture is also an essential aspect of tagging bats for individual identification and tracking studies. 6. Passive acoustic monitoring is a valuable non-invasive method for continuous monitoring of within-night, seasonal and between-year variation in the abundance of bat calls. These can be used as an index of variation in relative abundance within - but not between - bat species.
\end{abstract}

\section{Introduction}

Bats make species-specific calls for echo-location and social functions, enabling the presence of different species and changes in their calling activity to be monitored non-invasively by passive recording and identification of their calls. Bats do, however, vary their calls in relation to different habitats, functions, and the presence of other bats. This can result in difficulties of species recognition where the calls of one species are similar to the calls of another. As a result, for some species capture and examination in the hand (or even DNA confirmation) may be needed to identify individual bats to species level (Kuenzi \& Morrison 1998). Furthermore, different bat species emit different intensity or directionality of calls, and at different frequencies (e.g. Barataud 2015, Anderson \& Racey 1991, Goerlitz et al. 2010), with the inevitable result that some species' ultrasonic calls are easier to detect than others, leading to a bias in the species abundance being detected using acoustic methods. In spite of these issues, recording of acoustic activity can give a good indication of activity patterns (Beason et al. 2020).

Trapping also has inherent biases, as some species of bats are better at either avoiding traps or escaping from them than others (personal observations). Bats also differ in the height at which they fly above traps, which are typically set 1-4 m above ground level, and thus avoid them. Leon-Tapia and Hortelano-Moncada (2016) reported that in their study in Mexico, 12 species of bats were detected by using ultrasonic detectors, 
whereas only 5 species were trapped. Furthermore, trapping inevitably disturbs a bat's natural activity. Indeed, trapping on successive nights leads to reduced catches, indicating some alteration in behaviour (Kunz \& Brock 1975). Thus, this method cannot be utilised to observe activity accurately over small time periods, even were all species to be trapped. Given the limitations of each of these different approaches, both ultrasonic detectors and capture are needed to characterise local bat assemblages (Kunz et al. 2009).

Developments in bat detection and identification technology have enabled more detailed recording of bat echolocation calls, and the automated identification of the species emitting the call. Unfortunately, however, independent testing of these systems reveals variable effectiveness, with individual researchers' manual identification of calls from sonograms also showing imperfect species recognition (Clement et al. 2014, Russo \& Voigt 2016, Rydell et al. 2017). This is hardly surprising given the variability in call structure shown by individual species of bats in different habitats, and the inherent similarity between the calls of some species (Barataud 2015).

In Europe, species of bats within the Myotis genus (including Brandt's bats Myotis brandtii, Daubenton's bats Myotis daubentonii, Natterer's bats Myotis nattereri and whiskered batsMyotis mystacinus ), as well as other species such as the barbastelle bat Barbastella barbastellus and the brown long-eared bat Plecotus auritus, aggregate at certain sites in Autumn, in a poorly-understood social activity known as "Autumn swarming" (Fenton 1969, Parsons et al. 2003a \& b, Rivers et al. 2006, Glover et al. 2008). In swarming aggregations, bats appear to congregate primarily for the purpose of mating; sex ratios at swarming sites on any specific night are heavily biased towards males. Females attend swarming sites sporadically, which is believed to be in order to mate (Furmankiewicz et al. 2013, Parsons et al. 2003a \& b, Rivers et al. 2006, Glover et al. 2008, van Schaik et al. 2015). Bats may attend swarming aggregations from a wide geographical area; for example, Rivers et al. (2006) found that a swarming site for Natterer's bats had a catchment radius of up to $60 \mathrm{~km}$.

Autumn swarming in these species is usually focused around underground formations (Glover et al. 2008, Parsons et al. 2003b), with common features including a well-developed underground chamber, absence of water in the chamber, and shelter (e.g. vegetation) at the entrance. There is no correlation between swarming activity and the size of entrance opening (Glover et al. 2008). Parsons et al. (2003a) reported that peak swarming activity generally occurs 6-7 hours after sunset, is reduced by rain, and is positively correlated with ambient temperature. The swarming season for Myotis bats is late summer to early autumn, with Brandt's bats and Daubenton's bats swarming relatively early in this period, whilst Natterer's bats and whiskered bats swarm later in the season (Parsons et al. 2003a).

Studies of swarming bats typically involve capture of bats using mist nets or harp traps (Parsons et al. 2003a, Rivers et al. 2006, Glover et al. 2008) which is inevitably disturbing to the bats and hence can only be undertaken infrequently during any one swarming season. Trapping of bats is not a good measure of the absolute numbers present, with catch efficiencies typically being of the order of $5-20 \%$ of the total number of individuals using the site on that night (Altringham 2017). Furthermore, although individual adult males can stay at the swarming site for many nights (Altringham 2017, personal observations), turnover of individuals between nights and potential trap-shyness effects lead to recapture rates of males being only $0.5 \%$ or lower (Altringham 2017, Dekeukeleire 2017).

In addition to trapping and handling, swarming activity can be monitored much less intrusively using passive logging of bat ultrasonic calls. Glover et al. (2008) and Parsons et al. (2003a) report that there is a strong positive correlation between the number of bat echo location calls logged at swarming sites, and the numbers of bats caught. Records of bat activity in these previous studies only comprised the total number of bat passes (sequences of calls recorded by the bat detector), with no attempt to identify individual species of bat.

In the present study, the "Bat Classify" software (Scott and Altringham 2014) was used to investigate Myotis bat activity at a swarming site across the autumn swarming period, to determine whether the activity patterns of different species as measured by the classification software is consistent with the activity 
patterns as measured by trapping. The continual automated acoustic monitoring of bats across the active season provides much more detailed temporal information on bat activity than sporadic trapping sessions can. These combined methods were used to examine seasonal and overnight patterns of activity of each Myotis species present at a multi-species swarming site in the Wye Valley, on the border between Wales and England.

\section{Materials and Methods}

\section{Study area}

The study area was an area of broadleaved woodland to the North of Chepstow (Wales, UK; centred on latitude 51.6711, longitude -2.6862) on the steep sides of the River Wye valley. In the area are several natural cave formations and man-made openings in the predominantly limestone rock strata. One of these caves within the woodland (at latitude 51.67216, longitude -2.68493) has a small opening ( $600 \mathrm{x} 500 \mathrm{~mm}$ aperture) but extends a considerable distance downward (at least $50 \mathrm{~m}$ ). This cave, referred to as "Middle Earth" in the study by Davison \& Thomas (2017), has recently been shown to be a site used for swarming by multiple species of bats, including several Myotisspecies (personal observations).

\section{Measurement of bat activity}

A Titley Scientific Anabat Swift bat detector (https://www.titley-scientific.com) was deployed to monitor bat calling activity immediately outside the cave. It was set to record full spectrum echo location calls, at a $500 \mathrm{kHz}$ sampling frequency. The detector switched on automatically at 15 minutes before sunset and turned off 15 minutes after sunrise. Monitoring in 2017 was undertaken from 26 July to 29 October, with 14 of the 96 nights within this period being missed due to the equipment failing to activate. Monitoring in 2018 was undertaken from 23 March until 2 November; no nights of data capture were missed. In addition, a Titley Scientific Walkabout hand-held bat detector (https://www.titley-scientific.com) was used occasionally in the nearby woodland, recording at $500 \mathrm{kHz}$ in full spectrum.

Manual identification of echo location calls was undertaken by visual inspection of sonograms using "Anabat Insight" software (https://www.titley-scientific.com) by one observer (SPD), and automated analysis of calls was undertaken using "Bat Classify" software available at https://bitbucket.org/chrisscott/batclassify/downloads. This software was chosen as it reliably identifies the calls of most British woodland bats, including all Myotis species in the study area. This automated software does not attempt to differentiate between calls of whiskered and Brandt's bats, due to the similarity between the echo location calls of these two species. Only calls that the Bat Classify software identified to species level with $80 \%$ confidence levels or more were used for the purpose of this study.

Individual species within the Myotis genus may have different rates of correct species assignment, as some species may be easier for the software to recognise from their acoustic signature than others, and some species may more frequently give atypical calls at the swarming site as opposed to the calls that they make in their more usual habitat.

Video monitoring of entry and exit of bats at the cave entrance was undertaken on two occasions (28 August and 23 September 2017), using a Canon XA10 video recorder working in infra-red mode with an infra-red light source. Recording were made from 30 minutes before sunset to 3.5 hours after sunset. Numbers of bats entering and leaving the cave were noted.

In parallel with, and before this study, three years of bat echolocation data (2015 -2017) were collected at a nearby cave ("Hobbit Hole" 51.6707, -2.6864) and analysed using a Wildlife Acoustics SM3 Bat detector recording in "zero-crossing" mode (see Davison \& Thomas 2017). The resulting sonograms were analysed by visual inspection to identify bat calls to genus level.

\section{Meterolological data}

Hourly temperature and rainfall data for 2017 were obtained for the whole study period, from the daily Meteorological Office summaries available at www.metoffice.gov.uk/public/weather/observation/gcnjg1jby. 
For the analysis, rainfall was quantified as the percentage of the night (to the nearest $10 \%$ ) in which rain was detected. In 2017, ambient temperature at midnight (GMT) was the chosen nightly temperature used. For 2018, dusk temperature was also obtained from the Titley Anabat Swift bat detector, and as this proved to be a better predictor of bat activity than midnight temperature (see Results), dusk temperature was used in the analysis of 2018 data.

\section{Examination of bats in the hand.}

All bats caught for this study were trapped under a licence issued by Natural Resources Wales (licence no. 73106c:OTH:SRAB:2017). Catches took place on multiple nights during the swarming seasons of 2013 and 2018, in four different areas of the woodland around the focal cave ("Middle Earth"). All catches were made within $100 \mathrm{~m}$ of the cave entrance. Bats were only trapped at the cave entrance on two occasions, so as to minimise the possibility of bats in the traps echo locating and being detected by the bat detector at the cave entrance, thereby falsely elevating the number of calls recorded. Alternatively, the presence of the trap may have acted to keep bats away from the cave and therefore reduced the number of calls recorded. Trapping sessions ran from sunset until bat activity declined substantially (in the early hours of the morning). The exceptions were the nights of 22 September 2017 and 12 September 2018, when on both nights trapping terminated at 22.00 GMT even though activity at the time was high.

\section{Analysis of data}

Analyses were carried out using the statistical software "R" (version 3.2.3. R Development Core Team 2016), with methods following Thomas et al. (2017). The MASS package (Venables and Ripley 2002) and the mgcr package (Wood 2011) were used to implement for each species a negative binomial Generalised Additive Model (GAM) analysis of the number of echo location calls. Model selection was tailored to identify the detail of seasonal patterns (week-to-week variation), by choosing K-values (degree of non-linearity) that was higher than needed to minimise AIC. These models examined non-linear temporal (seasonal and/or overnight) variation in calling activity, and tested for the linear effects of both temperature and rainfall on activity. We used the contrast package (Kuhn 2016) to examine pairwise differences between specific weeks in the number of calls recorded. Capture data were analysed using a Chi-squared approach to test for sex-ratio differences for each Myotisspecies within and between years, and using negative binomial GAM models to examine seasonal and between-year variation in capture rate. Outputs of all GAM models are presented in the Appendix. Data files and R script files for running the analyses are archived in the Supplementary Materials.

\section{Results}

\section{The use of the study area by swarming Myotis bats}

The study area (encompassing multiple cave entrances) has recently been shown to be a swarming site for Myotis bats, as well as for lesser horseshoe bats Rhinolophus hipposideros (personal observations, Davison \& Thomas 2017). Analysis of zero crossing echolocation files recorded at a nearby cave ("Hobbit Hole") between 2015 and 2017, and analysed by visual inspection of the resulting sonograms, shows the considerable increase in Myotis bat activity in Spring and during the Autumn swarming season (Figure 1). The peak in the Spring is assumed to be when bats are emerging from hibernation in the caves. The timing of Autumn swarming at this cave (all Myotisspecies combined) varies slightly between the years, but in general lasts from late July until early October. In 2016 and 2017, Myotis activity during the Autumn swarming season exhibited a clear double-peak of activity, with a gap of 1-2 weeks between these peaks. In 2015, the swarming season started later and finished earlier than in the other two years, with less overall activity and a less evident double peak, with the primary peak in 2015 falling intermediate in date between the clear double peaks of the other two years.

Figure 1 : Seasonal variation in the activity of Myotisbats (all species combined) at "Hobbit Hole" cave in 2015-2017, measured as the number of zero-crossing files, and identified to genus level by visual inspection of the resulting sonograms. 


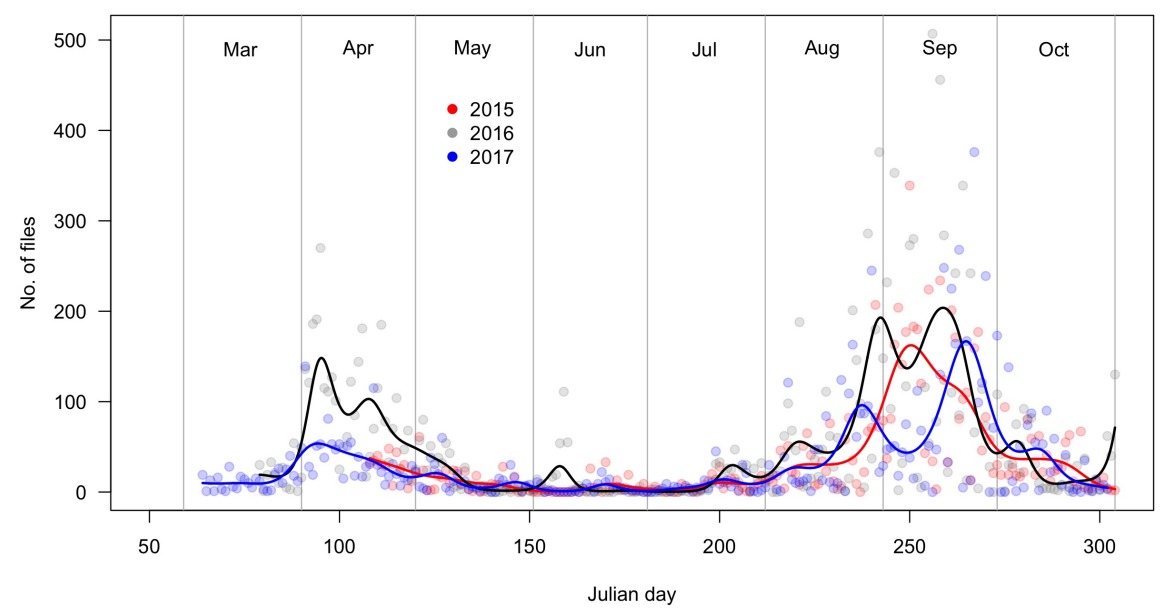

\section{Capture data}

Trapping of bats in woodland adjacent to the caves could only be undertaken on a few nights in each swarming season (to minimise disturbance). To obtain sufficient sample size for each taxon, catches were pooled for analysis across 2013-2018. Given that acoustic analysis (Fig 1a \& b) shows that the phenology of swarming shifts between years in terms of calendar date, this tends to broaden the apparent seasonal peak in capture rate when capture data are pooled across years, as in Figure 2. Overall, the calendar order of peak swarming activity for each species follows the same order as individually identifiable species detected acoustically (i.e. Daubenton's > Bechsteins > Natterer's). The captured sample of Myotis bats showed a strongly male-biased sex ratio, and this male-bias did not vary significantly between years (Table 3).

Table 1: Sex ratios among Myotis bats captured using mist nets and harp traps. Male : female ratios were compared using Fisher's exact test for sex-ratio bias (null hypothesis of 1:1), and for differences in sex ratio between 2017 and 2018 .

\begin{tabular}{|c|c|c|c|c|c|c|c|c|c|}
\hline Species & 2017 & 2017 & 2017 & 2018 & 2018 & 2018 & $\begin{array}{l}\text { Total } \\
2017- \\
18\end{array}$ & $\begin{array}{l}\text { Total } \\
2017- \\
18\end{array}$ & $\begin{array}{l}\text { Total } \\
2017- \\
18\end{array}$ \\
\hline & M:F & $\begin{array}{l}\% \\
\text { Male }\end{array}$ & $\mathbf{P}$ & M:F & $\begin{array}{l}\% \\
\text { Male }\end{array}$ & $\mathbf{P}$ & M:F & $\begin{array}{l}\% \\
\text { Male }\end{array}$ & $\mathbf{P}$ \\
\hline $\begin{array}{l}M . \\
\text { bech- } \\
\text { steinii }\end{array}$ & $19: 2$ & 90.5 & 0.006 & $21: 5$ & 80.8 & 0.040 & $40: 7$ & 85.1 & 0.0004 \\
\hline $\begin{array}{l}M . \\
\text { nattereri }\end{array}$ & $39: 9$ & 81.2 & 0.002 & $62: 5$ & 92.5 & $<0.0001$ & 101:14 & 87.8 & $<0.0001$ \\
\hline $\begin{array}{l}\text { M. } \\
\text { mystac- } \\
\text { inus }\end{array}$ & $35: 11$ & 76.1 & 0.020 & $17: 4$ & 81.0 & 0.050 & $52: 15$ & 77.6 & 0.001 \\
\hline $\begin{array}{l}M . \\
\text { brandtii }\end{array}$ & $4: 3$ & 74.3 & - & $1: 0$ & 100.0 & - & $5: 3$ & 62.5 & - \\
\hline
\end{tabular}




\begin{tabular}{|c|c|c|c|c|c|c|c|c|c|}
\hline Species & 2017 & 2017 & 2017 & 2018 & 2018 & 2018 & $\begin{array}{l}\text { Total } \\
2017- \\
18\end{array}$ & $\begin{array}{l}\text { Total } \\
2017- \\
18\end{array}$ & $\begin{array}{l}\text { Total } \\
2017- \\
18\end{array}$ \\
\hline $\begin{array}{l}M . \\
\text { dauben- } \\
\text { tonii }\end{array}$ & $33: 5$ & 86.8 & 0.001 & $13: 1$ & 92.9 & 0.030 & $46: 6$ & 88.5 & $<0.0001$ \\
\hline
\end{tabular}

Catches in 2017 and 2018, showed that the peak of swarming activity of Brandt's bat occurs earlier in the season than the peak swarming activity of whiskered bats (Figure 2) but at the cave itself, very little acoustic activity was detected that could be attributed to these two species which are hard to differentiate acoustically. It is unlikely that this was due to the limitations of the Bat Classify software, as in the adjacent woodland $12.6 \%$ of Anabat Walkabout's 87 files were identified by the Bat Classify software as either whiskered or Brandt's bats. This shows that the calls of these two species were detectable and successfully identified by the software in the woodland; hence there was probably a genuine lack of activity by these two species at the cave. The presence of clay on one wing of both a Daubenton's bat and a whiskered bat trapped early in the evening is highly suggestive of them having been roosting underground in the vicinity.

Figure 2: Catches of different Myotis species in 2013-18. Captures are plotted against calendar date, but in each year the timing of peak activity was different. 

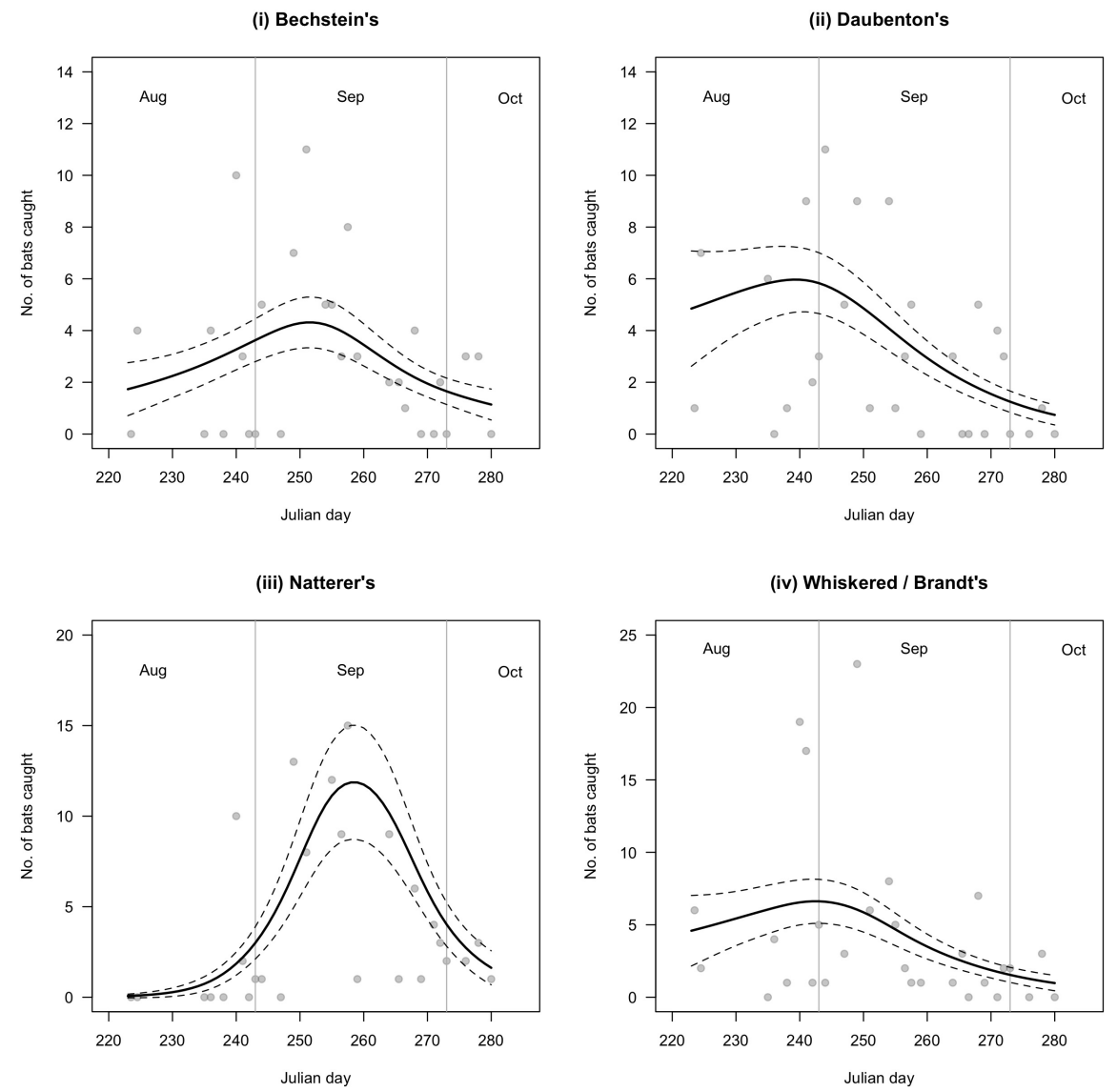

\section{Video monitoring of use of the cave by bats}

It was not possible to identify the bats to species-level using the video recordings, some were shown from analysis of simultaneously-recorded heterodyne sound files to have been Rhinolophus hipposideros (and therefore removed from the analysis), whereas others were Myotis species. Overall bat activity (number of entries and exits) detected by infra-red video monitoring on 23 September 2017 was greater than that recorded earlier on 28 August (Figure 3). On both dates, bats initially left the cave (where many had been roosting) following sunset. Bats later returned to the cave, many exhibiting "chase" sequences (one bat following another) which were visible on the infra-red video recordings. From $1.5-2$ hours after sunset there was a net influx of bats into the cave.

Figure 3 : Video-evidence of bats entering and leaving the focal cave on 28 August and 23 September 2017, from half an hour before dusk, until 3.5 hours after dusk. 


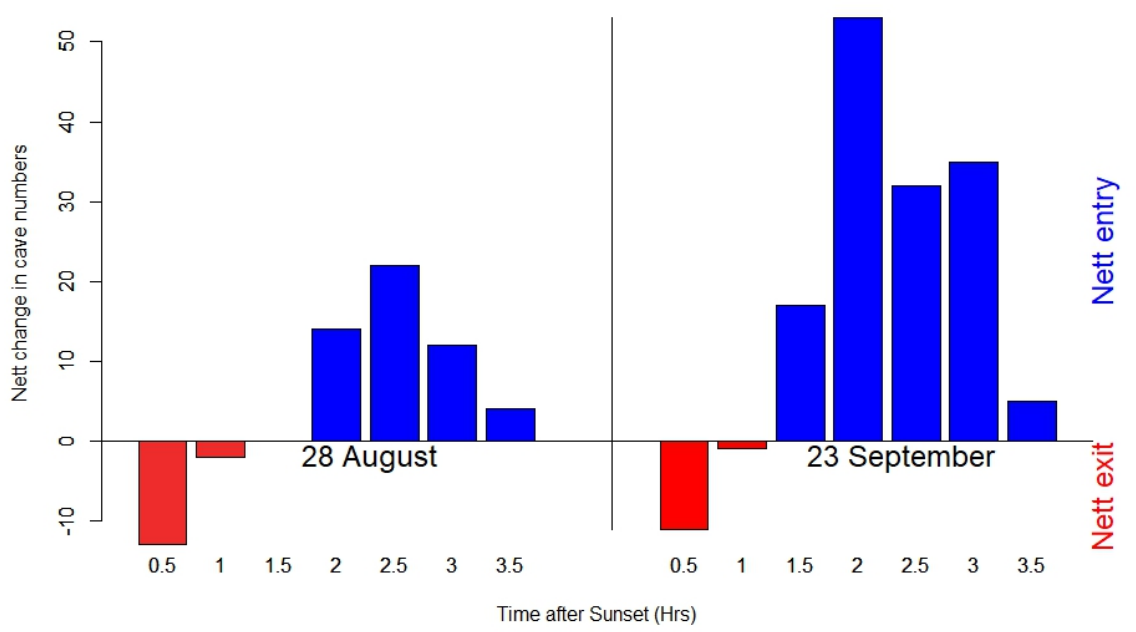

The effectiveness of the Bat Classify software

Different Myotis bat species have similar calls, which are often difficult to separate from - and may overlap with - the calls of otherMyotis species (Barataud 2015). A confidence level of $80 \%$ was selected for the automated identification of species using the Bat Classify software. Below the $80 \%$ confidence level, the Bat Classify software frequently gave more than one possible identification for the calls, whereas above the $80 \%$ level, too few call sequences were identified to allow a substantial sample size for statistical analysis. Even at this confidence level, many Myotis call sequences were not ascribed to an individual species. Indeed, only $27 \%$ of calls that were manually identified as Myotis type calls were assigned by the automated software to a particular Myotis species at the $80 \%$ confidence level or above. In contrast, for a more readily identifiable species, $96 \%$ of manually identifiable lesser horseshoe bat Rhinolophus hipposideros calls were assigned by the software to this species at the $80 \%$ confidence level or above.

\section{Results of Automated Acoustic Monitoring}

\section{a) Seasonal variation in activity}

Variation in acoustic activity during 2018 clearly shows species differences in seasonal patterns (Figure 4 panels iii-vi). The acoustic monitoring reveals that Daubenton's bat swarms earlier than Natterer's bat. Bechstein's bat had a much lower number of calls recorded (as would be expected from trapping records, and its lower acoustic amplitude; Barataud 2015), but is active across the Myotisswarming period. In the case of Brandt's/whiskered bats, which cannot be reliably separated by acoustic analysis, there are two main periods of activity; early and late in the swarming season. The catch data (Figure 2) show that Brandt's bat appears earlier in the season than whiskered bat, suggesting that the earlier acoustic peak in Figure 1b panel iv, is primarily composed of Brandt's bats and the later peak is primarily composed of whiskered bats. Figure 1b panel ii shows bimodal peaks of calling activity in 2018 for Natterer's bat. The pattern for other species is less clear, possibly due to the lower counts involved.

Figure 4: Seasonal variation in the activity of Myotisbats at "Middle Earth" cave in 2018. Panel (i) shows all Myotisspecies combined, detected as full spectrum sonograms and identified by automated identification to genus using the Bat Classify software. The temperature data shown in panel (ii) were obtained from the mouth of the cave at dusk, and the smoothed seasonal pattern (fitted using a GAM, $\mathrm{k}=6$ ), is shown in addition to the raw data. Seasonal activity patterns of individual taxa are shown in panels (iii) to (vi). 

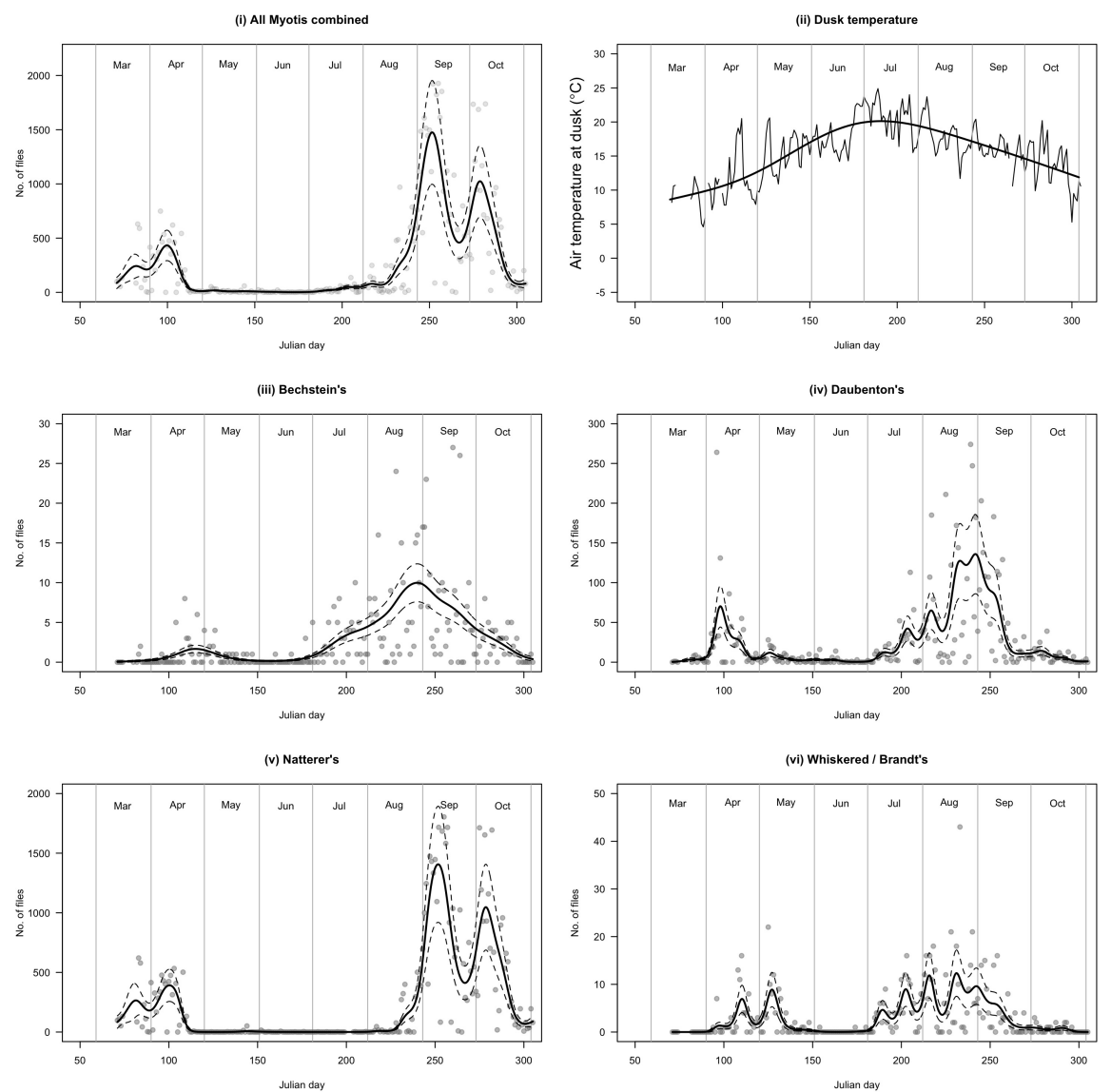

\section{b) Activity throughout the night}

The activity of different Myotis bat species, as measured by acoustic monitoring, varied both through the night, and through the season. Figure 5 shows nightly activity of Natterer's and Daubenton's bats - from dusk, for each week of significant activity. There were too few hourly data points for Brandt's/whiskered and Bechstein's to analyse in this way. Peak overnight activity for these two species varies slightly between weeks but occurs typically about 4 hours after dusk. Within the swarming period for each individual species there was a tendency for activity to continue further into the night as nights became longer later in the year.

Figure 5: Hourly activity of different Myotis species across the night, across the 2018 peak swarming period. The vertical lines indicate the changing night lengths throughout the study period, and the colours indicate respective weeks. (i) Overnight distribution of Myotis nattereri calls. (ii) Overnight distribution of Myotis daubentonii calls. Vertical lines and colours represent mean time of sunrise during each week of the study period. 
(i) Daubenton's

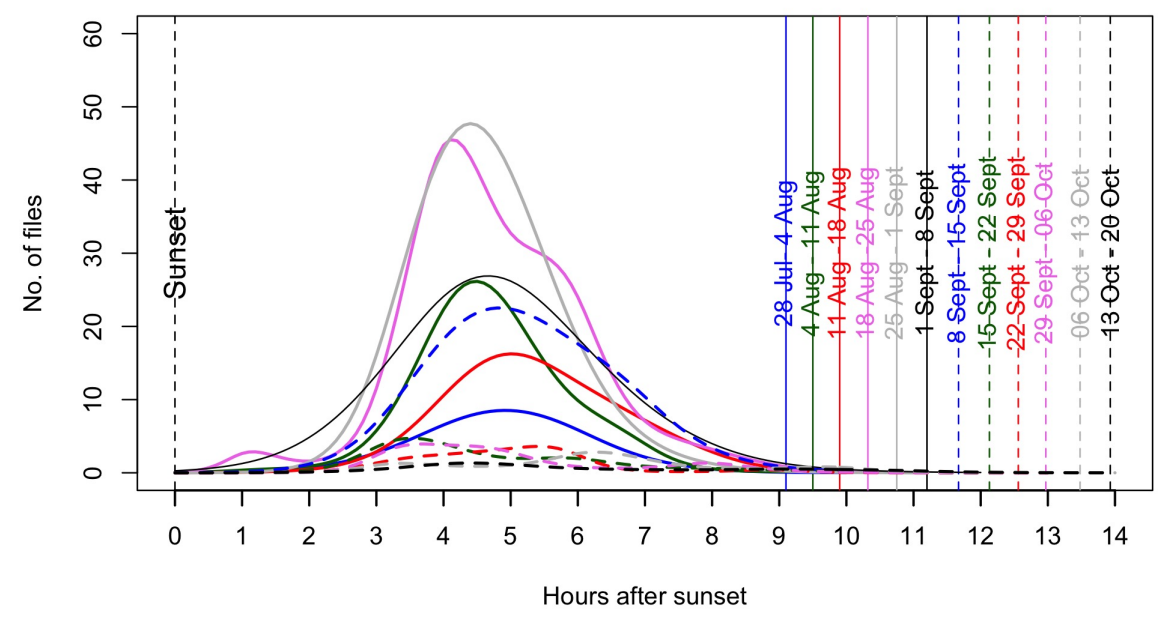

(ii) Natterer's

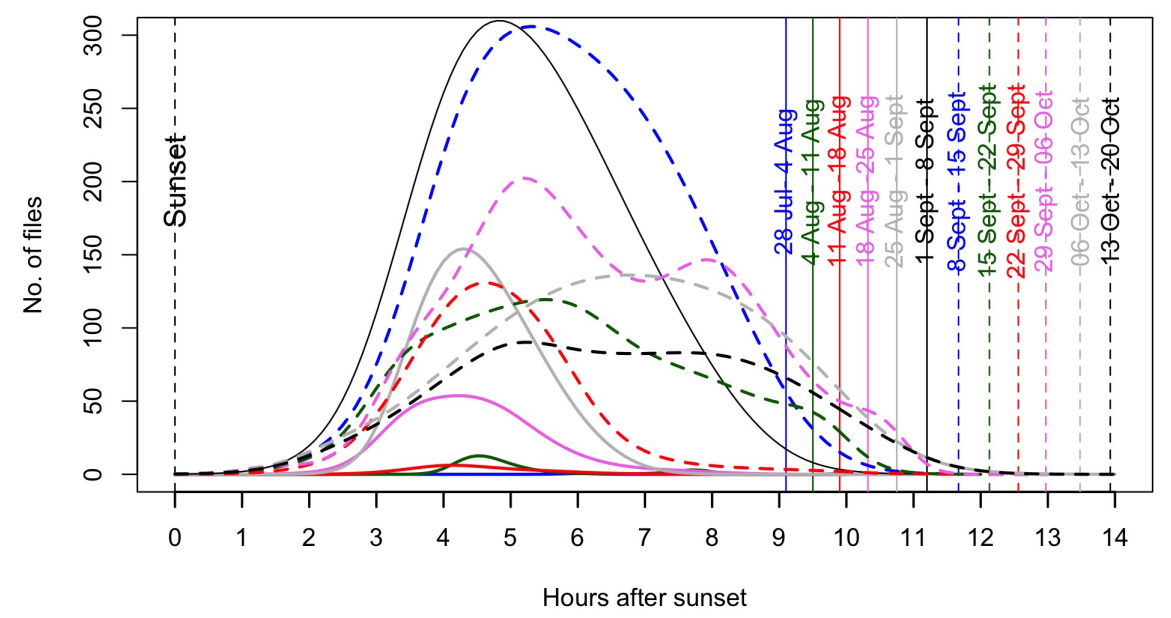

\section{Discussion}

Automatic analysis of bat echo location calls using Bat Classify yieldsMyotis species' activity patterns consistent with those gained from trapping. Furthermore, this information is available constantly throughout every night of the swarming season, whereas trapping is inevitably sporadic due to the disturbance caused to the bats and can only be undertaken infrequently during the swarming season. Both methods have their advantages and disadvantages. In the case of trapping, radio-tracking data for male bats suggests that they can learn to avoid traps within the swarming season (personal observations). This "trap-shyness" would affect the numbers captured on subsequent nights (see also Kunz \& Brock 1975) and impairs population estimation from data on captures and recaptures within the same year. Additionally, some species, demographic groups, or individuals, may be better at avoiding traps in the first place, leading to potential bias in population estimates.

Whilst acoustic monitoring is most unlikely to affect a bat's activity, different species have different intensities of call. This leads to a microphone detecting some species at greater distances than others, with consequential bias in comparing abundance between species by this method. Additionally, different species are likely to have different probabilities of identification from analysis of their echo location calls. Absolute activity 
levels of one species determined by this method cannot therefore be compared directly to those of another species. Absolute abundance is therefore unlikely to be measured effectively by either method, but both have their different advantages in determining changes in relative abundance within taxa, for example across the swarming season and across the night.

Capture gives the opportunity to identify bats in the hand, to note their sex, identify sex ratio biases (Table 1) and in the case of males, to record their reproductive status. In the case of females, it is usually possible to observe whether they have given previously birth, and sometimes whether the individual is a juvenile. In contrast, acoustic monitoring is of no use in determining sex or breeding status of the population. It is, however, useful in determining seasonal (nightly, Figure 4) and overnight (hourly, Figure 5) activity patterns, which have been shown in the present study to vary substantially throughout the swarming period. Video monitoring combined with acoustic detection adds an extra dimension to this information, by revealing that Myotis bats increasingly accumulate within the cave during the first part of the night, following an initial exodus at dusk (Figure 3).

Manually checking several thousand files of echo location data each night through multiple seasons would prove to be a challenge, hence automatic classification of the calls is the only practical way forward, even though neither approach is likely to be $100 \%$ accurate (Russo \& Voigt 2016, Rydell et al. 2017). Nevertheless, the aggregated automatically identified acoustic records correspond well with the capture data (Figures 2 and 4).

The notable bimodal peak in swarming activity of Myotis nattereri, shown for the first time in the present study (Figure 4), is unlikely to have been detected by sporadic trapping (cf. Figure 2). A possible explanation for this bimodality is that swarming has multiple functions; for example mating could account for the first peak, and pre-hibernation activity could account for the second peak -but this interpretation clearly needs further investigation. There is a suggestion of similar activity patterns for the other Myotisspecies, although the lower numbers recorded prevented a firm conclusion in these cases.

This study has demonstrated that the use of acoustic monitoring adds significantly to data obtained by catching bats at swarming periods, and for some research questions may provide sufficient or additional information without disturbing the animals. The automated acoustic identification software has its limitations in consistently identifying individual species' echo location calls, but, over the swarming season, patterns of activity shown by acoustic monitoring are remarkably consistent with those derived from trapping, but allow a far more detailed analysis of temporal (seasonal and overnight) variation. If the efficiency of the algorithms used to identify bats can be improved, this technique for quantifying seasonal variation in bat activity, including swarming activity, will become even more effective. Overall, neither trapping nor acoustic identification alone provide a fully comprehensive and accurate method of studying bat swarming behaviour, but the use of both methods, in parallel with additional approaches such as infra-red video monitoring of cave entrances, represents a powerful combined approach, providing a deeper understanding of bat behaviour than can be gained using either method individually.

\section{Acknowledgements}

The authors would like to thank National Resources Wales for access permissions and a licence to trap bats, N. Wynne-Hughes, S. Wadley, S. Ayling and L. Kergon for invaluable help in the field, and T. Hefin Jones for his valuable comments on an earlier draft of this paper.

\section{Author contributions}

SPD collected and processed all of the field data. SPD and RJT together analysed the processed data and co-wrote the manuscript.

\section{Data availability}

The datasets and $\mathrm{R}$ script files will be archived in the Dryad data repository.

\section{References}


Altringham, J. (2017). Presentation at Bat Conservation Trust Swarming Conference, 2-3 Sept 2017.

Anderson, E. \& Racey, P. A. (1991). Feeding behaviour of captive brown long-eared bats Plecotus auratus. Animal Behaviour, 42, 489-493.

Barataud, M. (2015). Acoustic Ecology of European Bats: Species Identification, Study of their Habitats and Foraging Behaviour. Biotope, Meze, Paris.

Beason, R. D., Riesch, R., \& Koricheva, J. (2020). Temporal Pass Plots: an intuitive method for visualising activity patterns of bats and other vocalising animals, Ecological Indicators, 113, 1-5.

Clement, M. J., Murray, K. L., Solick, D. L., \& Gruver, J. C. (2014). The effect of all libraries and acoustic filters on the identification of bat echolocation. Ecology and Evolution, 4(17), 3482-3493.

Davison, S. P. \& Thomas, R. J. (2017). Apparent spring swarming behaviour of Lesser Horseshoe bats (Rhinolophus hipposideros ). Journal of Bat Research and Conservation, 10(1). 10.14709/BarbJ.10.1.2017.03

Dekeukeleire, D. (2017). Presentation at Bat Conservation Trust Swarming Conference, 2-3 Sept 2017.

Dekeukeleire, D., Janssen, R., Haarsma, A. J., Bosch, T. \& Van Schaik, J. (2016). Swarming behaviour, catchment area and seasonal movement patterns of the Bechstein's bats: Implications for conservation. Acta Chiropterologica, 18(2), 349-358.

Fenton, M. B. (1969). Summer activity of Myotis lucifugus at hibernacula in Ontario and Quebec. Canadian Journal of Zoology, 47, 597-602.

Furmankiewicz, J., Duma, K., Manias, K. \& Borowiecz, M. (2013). Reproductive status and vocalisation in swarming bats Indicate a mating function of swarming and an extended mating function in Plecotus auritus . Acta Chiropterologica, 15(2), 371-385.

Glover, A. M. \& Altringham, J. D. (2008). Cave selection and use by swarming bat species. Biological Conservation, 141, 1493-1504.

Goerlitz, H. R., ter Hofstede, H. M., Zeale, M. R. K, Jones, G. \& Holderied, M. W. (2010). An aerial-Hawking bat uses stealth echolocation to counter moth hearing. Current Biology, 20, 1568-1572.

Kuenzi, A. J. \& Morrison, M. L. (1998). Detection of bats by mist nets and ultrasonic sensors. Wildlife Society Bulletin, 26, 307-311.

Kuhn, M. with contributions from S. Weston, J. Wing, J. Forester and T. Thaler. (2016). contrast: A Collection of Contrast Methods. R package version 0.21. https://CRAN.R-project.org/package=contrast

Kunz, T. H \& Brock, C. E. (1975). A comparison of mist nets and ultrasonic detectors for monitoring flight line activity of Bats. Journal of Mammology, 58, 309-315.

Kunz T. H., Hodgkison, R. \& Weise, C. D. (2009). In: Kunz, T. H. \& Parsons, S. Ecological Behavourial Methods for the Study of Bats, $2^{\text {nd }}$ Edition. John Hopkins University Press, Baltimore.

Leon-Tapia, M. A. \& Hortelano-Moncada, Y. (2016). Richness of insectivorous bats in a chaparral area in the municipality of Tecate, Baja California, Mexico. Revista Mexicana de Biodiversidad, 87, 1055-1061.

Parsons, K. N., Jones, G., \& Greenaway, F. (2003a). Swarming activity of temperate zone microchiropteran bats: effects of season, time of night, and weather conditions. Journal of Zoology, 261, 257-264.

Parsons, K. N., Jones, G., Davidson-Watts, I. \& Greenaway, F. (2003b). Swarming of bats at underground sites in Britain -Implications for conservation. Biological Conservation, 111, 63-70.

R Core Team 2016. R: A language and environment for statistical computing. R Foundation for Statistical Computing, Vienna, Austria. URL https://www.R-project.org/. 
Rivers, N. M., Butlin, R. K. \& Altringham, J. D. (2006). Autumn swarming behaviour of Natterer's bats in the UK: Population size, catchment area and dispersal. Biological Conservation, 127, 215-226.

Russo, D. \& Voigt, C. C. (2016). The use of automated identification of bat echolocation calls in acoustic monitoring: a cautionary note for sound analysis. Ecological Indicators, 66, 598-602.

Rydell, J., Nyman, S., Eklof, J., Jones G. \& Russo, D. (2017). Testing the performance of automated identification of bat echo location calls: a request for prudence. Ecological Indicators, 78, 416-420.

Scott, C. \& Altringham, J. (2014). Bat Classify Software. https://bitbucket.org/chrisscott/batclassify/downloads . Accessed July 2017.

Thomas, R. J. and the Guidebook Team. (2017). Data analysis with R statistical software: A guidebook for scientists. Eco-Explore, Caerphilly.

van Shaik, J., Janssen, R., Bosch, T., Haarsma, A., Dekker, J. J. A. \& Kranstauber, B. (2015). Bats swarm where they hibernate: compositional similarity between Autumn swarming and Winter hibernation assemblages at five underground sites. PLoS ONE, 10(7):e0130850. Doi:10.1371/ journal. Pone.0130850

Venables, W. N., \& Ripley, B. D. (2002). Modern Applied Statistics with R. Fourth Edition. Springer, New York.

Wood, S. N. (2011). Fast stable restricted maximum likelihood and marginal likelihood estimation of semi parametric generalised linear models. Journal of the Royal Statistical Society, B., 73(1), 3-36.

\section{Appendix materials}

Table A1: A Generalised Additive Model to explain variation in nightly abundance of different taxa of Myotis bats detected at Hobbit Hole cave in 2015-17 using automated acoustic monitoring. The model used a negative binomial error family $($ scale $=1)$ and a log-link function. Max $\mathrm{k}=40$, e.d.f. $=$ effective degrees of freedom. Overdispersion statistic $=1.061$, deviance explained $=61.7 \%$. The prediction plot from this model is shown in Figure 1.

\begin{tabular}{|c|c|c|c|c|c|c|}
\hline Independent variables & Category & d.f. / e.d.f. & Parameter estimate & $\mathrm{SE}$ & Test statistic & $\mathbf{P}$ \\
\hline Parametric terms & & & & & $\mathbf{Z}$ & \\
\hline Intercept & & 1 & 2.952 & 0.689 & 4.284 & $<0$ \\
\hline \multirow[t]{2}{*}{ Year $($ reference category $=2015)$} & 2016 & 1 & 0.030 & 0.698 & 0.043 & 0.966 \\
\hline & 2017 & 1 & -0.156 & 0.693 & -0.225 & 0.822 \\
\hline \multirow[t]{4}{*}{ Smoothed terms } & & & & & $\mathrm{Chi}^{2}$ & \\
\hline & J.day x 2015 & 18.94 & & & 278.4 & $<0$ \\
\hline & J.day x 2016 & 28.23 & & & 429.5 & $<0$ \\
\hline & J.day x 2017 & 27.29 & & & 292.2 & $<0.00$ \\
\hline
\end{tabular}

Table A2: Generalised Additive Models to explain variation in nightly abundance of different taxa of Myotis bats captured at Middle Earth cave in 2018. Each model used a negative binomial error family (scale = 1) and a $\log$-link function. Max $\mathrm{k}=40$, e.d.f. $=$ effective degrees of freedom.

1. Bechstein's bat. Overdispersion statistic $=1.907$, deviance explained $=15.9 \%$. The prediction plot from this model is shown in Figure 2(i).

2. Daubenton's bat. Overdispersion statistic $=1.922$, deviance explained $=26.9 \%$. The prediction plot from this model is shown in Figure 2(ii).

3. Natterer's bat. Overdispersion statistic $=1.673$, deviance explained $=45.9 \%$. The prediction plot from this model is shown in Figure 2(iii).

4. Brandt's / Whiskered bat. Overdispersion statistic $=1.642$, deviance explained $=25.5 \%$. The prediction plot from this model is shown in Figure 2(iv). 


\begin{tabular}{|c|c|c|c|c|c|c|}
\hline Taxon & $\begin{array}{l}\text { Independent } \\
\text { variables }\end{array}$ & d.f. / e.d.f. & $\begin{array}{l}\text { Parameter } \\
\text { estimate }\end{array}$ & SE & $\begin{array}{l}\text { Test } \\
\text { statistic }\end{array}$ & $\mathbf{P}$ \\
\hline \multirow{2}{*}{$\begin{array}{l}\text { i) } \\
\text { Bechstein's } \\
\text { bat }\end{array}$} & $\begin{array}{l}\text { Parametric } \\
\text { term }\end{array}$ & & & & $\mathbf{Z}$ & \\
\hline & $\begin{array}{l}\text { Intercept } \\
\text { Smoothed } \\
\text { term }\end{array}$ & 1 & 0.982 & 0.159 & $\begin{array}{l}6.175 \\
\text { Chi }^{2}\end{array}$ & $<0.0001$ \\
\hline \multirow{2}{*}{$\begin{array}{l}\text { ii) } \\
\text { Dauben- } \\
\text { ton's } \\
\text { bat }\end{array}$} & $\begin{array}{l}\text { Julian day } \\
\text { Parametric } \\
\text { term }\end{array}$ & 2.421 & & & $\begin{array}{l}6.529 \\
\mathbf{Z}\end{array}$ & 0.0892 \\
\hline & $\begin{array}{l}\text { Intercept } \\
\text { Smoothed } \\
\text { term }\end{array}$ & 1 & 1.116 & 0.160 & $\begin{array}{l}6.991 \\
\mathbf{C h i}^{2}\end{array}$ & $<0.0001$ \\
\hline \multirow{2}{*}{$\begin{array}{l}\text { iii) } \\
\text { Natterer's } \\
\text { bat }\end{array}$} & $\begin{array}{l}\text { Julian day } \\
\text { Parametric } \\
\text { term }\end{array}$ & 2.120 & & & $\begin{array}{l}14.870 \\
\mathbf{Z}\end{array}$ & 0.00133 \\
\hline & $\begin{array}{l}\text { Intercept } \\
\text { Smoothed } \\
\text { term }\end{array}$ & 1 & 1.998 & 0.203 & $\begin{array}{l}5.914 \\
\mathbf{C h i}^{2}\end{array}$ & $<0.0001$ \\
\hline \multirow{3}{*}{$\begin{array}{l}\text { iv) Brandt's } \\
\text { / Whiskered } \\
\text { bat }\end{array}$} & $\begin{array}{l}\text { Julian day } \\
\text { Parametric } \\
\text { term }\end{array}$ & 2.795 & & & $\begin{array}{l}25.040 \\
\mathbf{Z}\end{array}$ & $<0.0001$ \\
\hline & $\begin{array}{l}\text { Intercept } \\
\text { Smoothed } \\
\text { term }\end{array}$ & 1 & 1.266 & 0.170 & $\begin{array}{l}7.438 \\
\text { Chi }^{2}\end{array}$ & $<0.0001$ \\
\hline & Julian day & 2.199 & & & 0.006 & \\
\hline
\end{tabular}

Table A3: A Generalised Additive Models to explain variation in nightly activity of different taxa of Myotis bats (all taxa combined) at Middle Earth cave in 2018. The model used a negative binomial error family $($ scale $=1)$ and a $\log$-link function. Max $\mathrm{k}=40$, e.d.f. = effective degrees of freedom. Overdispersion statistic $=1.343$, deviance explained $=69.6 \%$. The prediction plot from this model is shown in Figure 4 , panel (i).

\begin{tabular}{lllllll}
\hline Independent variables & Category & d.f. / e.d.f. & Parameter estimate & SE & Test statistic & P \\
\hline $\begin{array}{lllllll}\text { Parametric term } \\
\text { Intercept }\end{array}$ & 1 & 4.205 & 0.0688 & 61.15 & $<0.0001$ \\
Smoothed term & & & & & $\mathbf{C h i}^{2}$ & \\
& J.day & 23.94 & & 785.6 & $<0.0001$ \\
\hline
\end{tabular}

Table A4: A Generalised Additive Model to explain variation in nightly abundance of different taxa of Myotis bats detected at Middle Earth cave in 2018, using automated acoustic monitoring. The model used a negative binomial error family $($ scale $=1)$ and a log-link function. Max $\mathrm{k}=40$, e.d.f. $=$ effective degrees of freedom. Overdispersion statistic $=1.208$, deviance explained $=82 \%$. The prediction plot from this model 
is shown in Figure 4 (panels iii-vi).

\begin{tabular}{|c|c|c|c|}
\hline Independent variables & Category & d.f. / e.d.f. & Parameter estimate \\
\hline \multicolumn{4}{|l|}{ Parametric terms } \\
\hline Intercept & & 1 & 0.322 \\
\hline \multirow[t]{3}{*}{ Species (reference category = Bechstein's) } & Daubenton's & 1 & 1.985 \\
\hline & Natterer's & 1 & 2.640 \\
\hline & Whiskered/Brandt's & 1 & -0.631 \\
\hline \multicolumn{4}{|l|}{ Smoothed terms } \\
\hline & Julian day x Bechstein's & 10.17 & \\
\hline & Julian day x Daubenton's & 29.95 & \\
\hline & Julian day x Natterer's & 25.51 & \\
\hline & Julian day x Whiskered /Brandt's & 26.95 & \\
\hline
\end{tabular}

Table A5: Generalised Additive Model to explain variation in hourly abundance of Daubenton's bats , detected at Middle Earth cave in 2018, using automated acoustic monitoring. The model used a negative binomial error family and a log-link function. The prediction plot from this model is shown in Figure 5 (i). Max $\mathrm{k}=15$, e.d.f. $=$ effective degrees of freedom. Overdispersion statistic $=0.974$, deviance explained $=$ $71.6 \%$.

\begin{tabular}{|c|c|c|c|c|c|}
\hline Independent variables & Category & d.f. / e.d.f. & Parameter estimate & SE & Test statistic \\
\hline Parametric terms & & d.f. & & & $\mathbf{Z}$ \\
\hline Intercept & & 1 & -0.739 & 0.573 & -1.290 \\
\hline \multirow[t]{13}{*}{ Week (reference category = Week 5) } & Week 1 & 1 & -0.713 & 0.816 & -0.873 \\
\hline & Week 2 & 1 & +1.565 & 1.035 & 1.512 \\
\hline & Week 3 & 1 & -0.089 & 0.770 & -0.116 \\
\hline & Week 4 & 1 & +0.777 & 1.434 & 0.542 \\
\hline & Week 6 & 1 & +1.499 & 0.613 & 2.446 \\
\hline & Week 7 & 1 & +0.806 & 0.638 & 1.264 \\
\hline & Week 8 & 1 & -0.897 & 0.835 & -1.074 \\
\hline & Week 9 & 1 & -2.054 & 1.538 & -1.336 \\
\hline & Week 10 & 1 & -1.729 & 1.176 & -1.470 \\
\hline & Week 11 & 1 & -0.993 & 0.948 & -1.047 \\
\hline & Week 12 & 1 & -0.838 & 0.688 & -1.219 \\
\hline & Week 13 & 1 & -4.878 & 2.998 & -1.627 \\
\hline & Week 14 & 1 & -9.841 & 22.635 & -0.435 \\
\hline \multirow[t]{14}{*}{ Smoothed terms } & & e.d.f & & & $\mathrm{Chi}^{2}$ \\
\hline & Hour x Week 1 & 3.178 & & & 56.091 \\
\hline & Hour x Week 2 & 7.232 & & & 100.614 \\
\hline & Hour x Week 3 & 3.980 & & & 68.257 \\
\hline & Hour x Week 4 & 8.995 & & & 104.527 \\
\hline & Hour x Week 5 & 4.330 & & & 118.014 \\
\hline & Hour x Week 6 & 3.518 & & & 109.619 \\
\hline & Hour x Week 7 & 4.472 & & & 105.544 \\
\hline & Hour x Week 8 & 6.120 & & & 34.072 \\
\hline & Hour x Week 9 & 6.782 & & & 31.353 \\
\hline & Hour x Week 10 & 6.428 & & & 26.687 \\
\hline & Hour x Week 11 & 7.620 & & & 21.382 \\
\hline & Hour x Week 12 & 5.053 & & & 16.567 \\
\hline & Hour x Week 13 & 3.204 & & & 10.979 \\
\hline
\end{tabular}




\begin{tabular}{llllll}
\hline Independent variables & Category & d.f. / e.d.f. & Parameter estimate & SE & Test statistic \\
\hline & Hour x Week 14 & 4.809 & & 2.988 \\
\hline
\end{tabular}

Table A6: Generalised Additive Model to explain variation in hourly abundance of Natterer's bats , detected at Middle Earth cave in 2018, using automated acoustic monitoring. The model used a negative binomial error family and a log-link function. The prediction plot from this model is shown in Figure 5 (ii). Max $\mathrm{k}=15$, e.d.f. $=$ effective degrees of freedom. Overdispersion statistic $=1.248$, deviance explained $=$ $77.2 \%$.

\begin{tabular}{|c|c|c|c|c|c|}
\hline Independent variables & Category & d.f. / e.d.f. & Parameter estimate & SE & Test statistic \\
\hline Parametric terms & & d.f. & & & $\mathbf{Z}$ \\
\hline Intercept & & 1 & 2.5575 & 0.221 & 11.576 \\
\hline \multirow[t]{13}{*}{ Week (reference category $=$ Week 7 ) } & Week 1 & 1 & -20.058 & 2.230 & -0.393 \\
\hline & Week 2 & 1 & -12.048 & 27.384 & -3.325 \\
\hline & Week 3 & 1 & -6.223 & 1.872 & -3.325 \\
\hline & Week 4 & 1 & -3.967 & 1.193 & -3.326 \\
\hline & Week 5 & 1 & -6.387 & 2.230 & -2.865 \\
\hline & Week 6 & 1 & -0.473 & 0.340 & -1.393 \\
\hline & Week 8 & 1 & -0.658 & 0.391 & -1.685 \\
\hline & Week 9 & 1 & -1.439 & 0.349 & -4.121 \\
\hline & Week 10 & 1 & -0.412 & 0.522 & -0.789 \\
\hline & Week 11 & 1 & 0.438 & 0.253 & 1.736 \\
\hline & Week 12 & 1 & 0.153 & 0.256 & 0.597 \\
\hline & Week 13 & 1 & -1.574 & 0.273 & -5.777 \\
\hline & Week 14 & 1 & -1.744 & 0.277 & -6.302 \\
\hline \multirow[t]{15}{*}{ Smoothed terms } & & e.d.f & & & $\mathrm{Chi}^{2}$ \\
\hline & Hour x Week 1 & 4.240 & & & 2.179 \\
\hline & Hour x Week 2 & 7.675 & & & 24.682 \\
\hline & Hour x Week 3 & 4.042 & & & 33.654 \\
\hline & Hour x Week 4 & 5.640 & & & 111.238 \\
\hline & Hour x Week 5 & 4.600 & & & 126.809 \\
\hline & Hour x Week 6 & 4.874 & & & 253.107 \\
\hline & Hour x Week 7 & 6.309 & & & 265.450 \\
\hline & Hour x Week 8 & 7.810 & & & 162.098 \\
\hline & Hour x Week 9 & 6.160 & & & 198.349 \\
\hline & Hour x Week 10 & 10.781 & & & 140.149 \\
\hline & Hour x Week 11 & 6.049 & & & 212.760 \\
\hline & Hour x Week 12 & 6.561 & & & 178.182 \\
\hline & Hour x Week 13 & 5.311 & & & 127.143 \\
\hline & Hour x Week 14 & 7.562 & & & 79.144 \\
\hline
\end{tabular}

